\title{
EXPERIENCIAS EN LA APLICACIÓN DE LA EDUCACIÓN AMBIENTAL COMO HERRAMIEN- TA PARA LA ADAPTACIÓN AL CAMBIO CLIMÁTICO EN ESPACIOS COMUNITARIOS, EN HOLGUÍN-CUBA
}

\section{Experiences in the implementation of environmental education as a tool for community adaptation to climate change in Holguin-Cuba.}

\author{
Olga Alicia Gallardo Milanés \\ Universidad de Holguín, Holguín, Provincia de Holguín, Cuba \\ oaliciagallardo2013@gmail.com
}

Artigo recebido em 01/10/2013 e aceito para publicação em 03/12/2013.

RESUMEN: El cambio climático afecta las condiciones de vida de la población al generar, impactos negativos en los espacios comunitarios: el incremento de las temperaturas, el aumento en la intensidad y frecuencia de los eventos meteorológicos extremos y otros efectos, provoca daños en lo económico, político, ambiental y social. Estas afectaciones pueden ser manejadas por una adaptación efectiva a través de la educación ambiental, concebida como una herramienta para preparar a las comunidades ante el cambio climático. Las acciones de educación ambiental estuvieron dirigidas a transferir conocimientos contextualizados con el propósito de desarrollar capacidades en los actores comunitarios, estas acciones promovieron el intercambio de saberes y la protección del ecosistema. En la investigación se utilizaron básicamente métodos cualitativos, combinándose enfoques metodológicos a partir de los diversos escenarios y procesos estudiados. Todo lo anteriormente expuesto se hizo en función de la interpretación de la realidad y el discurso de los actores involucrados de la ciudad de Holguín. Los resultados demuestran que la educación ambiental contribuye a la concientización de la población y motiva a los participantes a realizar transformaciones en sus comunidades.

palabras-claves: Educación ambiental, Comunidad, Vulnerabilidad, Adaptación, Cambio Climático.

ABSTRACT: Climate change affects the living conditions of the population, generating negative impacts on community areas. The rising temperatures, increased intensity and frequency of extreme weather events and other effects, damage the economy, politics, environment and society. These effects can be controlled by an effective adaptation, is environmental education as a tool to prepare communities to climate change. The actions of environmental education aimed at transferring contextualized knowledge in order to build capacity in community stakeholders, those actions promoted the exchange of knowledge and ecosystem protection. The research was basically done with qualitative methods, combining methodological approaches from the different scenarios and processes studied. The above mentioned was carried out in relation to the interpretation of reality and discourse of the actors involved in the city of Holguin. The results show that environmental education contributes to arise awareness on the population and encourages the participants to make changes in their communities.

keywords: Environmental education, Community, Vulnerability, Adaptation, Change climatic. 
Experiencias en la aplicación de la educación ambiental como herramienta para la adaptación al cambio climático en espacios comunitarios, en Holguín-Cuba

Olga Alicia Gallardo Milanés

\section{INTRODUCCIÓN}

El cambio climático presenta nuevos riesgos y mayores desafíos para el desarrollo sostenible, por el incremento e intensidad de los fenómenos meteorológicos extremos. La adaptación requiere desarrollar nuevas capacidades que permitan implementar estrategias efectivas, particularmente en el ámbito local. En este contexto, se requiere ser proactivos en la prevención de riesgos y la reducción de vulnerabilidades. Además es necesario conocer las fortalezas y debilidades de las comunidades para lograr una mejor planificación.

En la misma perspectiva Levin $\mathrm{T}$ y Encinas $\mathrm{C}$ (2008) consideran que por los efectos del cambio climático se requerirá desarrollar nuevas capacidades en el nivel local, construyendo sobre las ya existentes y probadas a ese nivel. En especial, será necesario dejar atrás el afán de centralización y sectorialización de los programas que no dan cuenta de las diversidades entre localidades en todo orden: ecosistémicas, culturales, base económica, geografía, clima, entre otras.

La educación ambiental es una herramienta para lograr la concientización de la población: contribuye a cambiar concepciones y costumbres en relación al ambiente, permite comprender las relaciones entre la sociedad y la naturaleza, estimula a intervenir en los problemas ambientales. Por lo tanto es un medio eficaz para preparar a las personas para una adaptación efectiva ante el cambio climático. Sin embargo, la experiencia de los últimos 30 años ha mostrado que la educación ambiental o la educación para la sustentabilidad, ha enfrentado diversos obstáculos, debilidades y retos en su camino de transformación hacia una educación participativa que pueda contribuir a cambios sociales y culturales, Dieleman H y Juárez M (2008).

Al preparar a las personas para la adaptación el cambio climático, se hace necesario lograr una participación efectiva de los actores comunitarios, darles protagonismo en el proceso, enseñarles a identificar las vulnerabilidades en sus propios medios de vida, definir las áreas prioritarias de atención, determinarlas opciones potenciales de reducción del riesgo, movilizar recursos disponibles y utilizar los conocimientos tradicionales.

En Cuba la educación ambiental en espacios comunitarios es realizada sin un currículo predeterminado, se consideran las características del lugar de in- tervención. El debate sobre los problemas ambientales favorece el desarrollo de un pensamiento crítico y de responsabilidad individual. La formación de capacidades basada en los valores humanos, se expresa en comportamiento personal de respeto, armonía con la naturaleza y compromiso de lograr acciones individuales y colectivas que favorezca el entorno.

La experiencia que se presenta se desarrolló en Holguín, capital de la provincia y municipio de igual nombre, ubicada en la porción central de la región norte del oriente del país, en un valle entre 100 y 120 m sobre el nivel medio del mar. En el año 2004, ocurrió un evento de sequía severo, colapsaron los tres embalses que suministran agua a la ciudad, los meteorólogos consideraron el período 2004-2005 como el más seco de los últimos 104 años. Ante esta situación se inició la puesta en práctica de un programa de educación ambiental para la adaptación al cambio climático en comunidades de la ciudad, pues según el grupo de clima del Centro Provincial de Meteorología, este fenómeno se observaba cada vez con más frecuencia.

Con el objetivo de preparar a la población ante las afectaciones del cambio climático, se implementó un programa de educación ambiental en las comunidades: Alcides Pino, Distrito Lenin y El Llano, en ellas se formaron capacidades para mejorar las dimensiones ambiental y sociocultural a través del enfoque de adaptación, pues este reconoce que las personas deben ser empoderadas, para transformar y garantizar sus medios de vidas, a través del diálogo dentro de las comunidades, promoviendo actividades que generen resiliencia.

\section{METODOLOGÍA}

En el proceso investigativo se emplearon métodos cualitativos, como la investigación acción, la observación participante y técnicas participativas, el estudio se estructuró en cuatro etapas. La primera etapa consistió en el diagnóstico y la caracterización socioambiental de las comunidades. En esta etapa, se aplicó la herramienta del análisis ciudadano de la situación ambiental local de ROLAC-UNHABITAT, (2011), una técnica para determinar, desde el punto de vista de los integrantes de una comunidad o localidad, sus problemas medioambientales y del hábitat, sus 
propuestas de soluciones y demandas para lograr su desarrollo de manera integral. Asimismo se efectuó un estudio de percepción de los diferentes actores, y se valoró el compromiso y la motivación. Además se aplicó la matiz de vulnerabilidad y capacidad para identificar las debilidades y fortalezas de las comunidades.

La segunda etapa fue dedicada a la socialización de conocimientos: la formación ambiental tuvo como eje fundamental la concepción de educación popular, como proceso continuo y sistemático que implica momentos de estudio y reflexión sobre la práctica, es repasar la realidad para lograr nuevos niveles de comprensión, si se quiere transformar la misma se realizará dentro de ella participando. Se confió en la capacidad de trasmitir conocimientos a través de los promotores ambientales, facilitando el protagonismo de los niños, niñas, jóvenes y demás miembros de la comunidad. En la tercera etapa se efectuaron acciones de recuperación del medio ambiente, las mismas estuvieron dirigidas a desarrollar habilidades, a aprender haciendo. La cuarta etapa se dedicó a la evaluación, que permitió la retroalimentación y el ajuste de cada una de las acciones pues fue efectuada de manera simultánea en cada una de las etapas ya mencionadas.

\section{LA EDUCACIÓN AMBIENTAL COMO HERRA- MIENTA PARA LAADAPTACIÓN AL CAMBIO CLIMÁTICO}

El cambio climático impone un nuevo reto para los países en desarrollo, pero particularmente son las comunidades locales, quienes deberán asumir nuevos enfoques, que les permita tomar decisiones en un escenario más dinámico y complejo. $\mathrm{Al}$ hacerse evidente la necesidad de encontrar respuestas a las vulnerabilidades existentes en los espacios comunitarios, a mediados de la década de los años 90 comienza a trabajarse el enfoque que se conoce como adaptación basada en comunidades, según la Secretaria de Ambiente y Desarrollo Sustentable de Argentina (2010), la característica distintiva de este es la participación de los actores, con miras a la reducción de riesgos.

El Panel Intergubernamental sobre Cambio Climático, define al cambio climático como cualquier cambio en el transcurso del tiempo, ya sea por su variabilidad natural, o como resultado de la actividad humana y expone además que la adaptación es el ajuste en los sistemas naturales o humanos, como respuestas a impactos climáticos actuales o futuros, que reduce el daño causado y que potencia las oportunidades benéficas, Grupo Intergubernamental de expertos sobre Cambio Climático (2007).

La adaptación al cambio climático es un proceso que requiere del compromiso y de una amplia gama de participantes y por consiguiente el fortalecimiento de la capacidad de la sociedad civil local y de las instituciones del gobierno, para que puedan apoyar más eficazmente, los esfuerzos que se desarrollan desde la comunidad, los hogares y los individuos. La adaptación se fundamenta también en el reconocimiento que las personas deben ser empoderadas para trasformar y garantizar sus derechos y medios de vida (CARE, 2010).

En armonía con lo expresado por Aldunce et al (2008) en relación a que la presencia de impactos producidos por el clima y la capacidad adaptativa, no se presentan en forma simultánea, pues en general los sistemas, requieren tiempo para adecuarse a los impactos de algún evento climático adverso, por lo que la reducción de la vulnerabilidad tampoco es paralela. Si las medidas de adaptación son anticipadas o planeadas, antes del evento climático adverso, el sistema habrá disminuido su vulnerabilidad a este evento antes de que se presente.

Adaptase a el cambio climático, significa manejar adecuadamente los riesgos, implementado programas o estrategias que protejan a los seres humanos y sus medios de vida. El PNUD (2008) se pronuncia porque los programas de adaptación incluyan cuatro componentes: Información para la planificación efectiva, infraestructura a prueba de eventos climáticos, seguros para gestionar el riesgo social y reducir la pobreza e instituciones para la gestión de los riesgos de desastres.

Varias acciones de adaptación, que hoy se proponen ante el cambio climático coinciden con las medidas que tradicionalmente ha planteado la gestión del riesgo. Algunas comunidades están en condiciones de utilizar conocimientos y experiencias para reducir los daños y promover el desarrollo, en este proceso, es preciso manejar información científico técnica y socioeconómica relevante para estos contextos, lo que permitirá comprender el riesgo que supone el cambio climático, provocado por las actividades humanas, sus posibles repercusiones y las posibilidades de 
Experiencias en la aplicación de la educación ambiental como herramienta para la adaptación al cambio climático en espacios comunitarios, en Holguín-Cuba

Olga Alicia Gallardo Milanés

adaptación o atenuación del mismo (COMUNIDAD ANDINA, 2009).

La educación juega un papel importante porque permite llegar a toda la población, contribuye a incorporar nuevos saberes y generar información sobre escenarios climáticos. La educación ambiental debe ser permanente en el tiempo y siempre estar disponible para los actores involucrados en el proceso. Debe ser gradual y ofrecer conceptos, contenidos y metodologías, así como herramientas básicas, que les permita a los comunitarios trabajar adecuadamente la adaptación. Educar para el cambio climático significa movilizar actores con capacidad para incorporar nuevos saberes, aprender haciendo y saber ser, pues la educación es fuente de transformación humana, es un instrumento para enfrentar desafíos como la crisis ambiental. En este sentido Sfeir-Younis (2009) significa que la mayoría de los debates nacionales e internacionales abrazan la necesidad de cambiar o intensificar los procesos educativos, por los problemas ambientales, de inseguridad entre otros, apunta que esta puede ser una solución.

La educación que se invierta en la adaptación al cambio climático, contribuye al desarrollo sostenible, pues se trata de empoderar a los actores para disminuir o mitigar los problemas ambientales, con iniciativas sustentables, donde la espiritualidad transformadora, combine lo individual y lo colectivo. La educación ambiental para la adaptación al cambio climático, debe cumplir los siguientes principios:

- Educación ajustada al contexto, considerando las particularidades de la comunidad en la que se va a desarrollar, orientada a satisfacer las necesidades de los grupos involucrados.

- La participación como eje fundamental del proceso formativo, donde se estimula el aprendizaje colaborativo, el diálogo de saberes y la crítica en función de cambiar la realidad.

- La combinación del conocimiento formal con la experiencia basada en acciones. Donde las acciones se realizan con el propósito de trasformar los espacios comunitarios a través de la solución o mitigación de los problemas ambientales.

- El desarrollo de habilidades que permitan interpretar la realidad y valorar el entono desde el pensamiento divergente.
- Fomentar la ética, sustentada en valores de solidaridad, humanismo, respeto, compromiso y corresponsabilidad.

\section{RESULTADOS Y DISCUSIÓN}

\section{Experiencias en la implementación de la educación ambiental para la adaptación al cambio climático}

El programa de educación ambiental implementado se caracterizó por iniciar con un diagnóstico en las comunidades, con el propósito de saber si los diferentes actores de estos espacios conocían los riesgos climáticos y las habilidades desarrolladas para enfrentar la sequía, si habían efectuado acciones o constituido alguna organización para afrontar los efectos de este fenómeno, que infraestructura física o natural existía y con qué recursos contaban.

Los resultados del diagnóstico revelaron que las personas no conocían los riesgos climáticos pues se entrevistó al $60 \%$ de la población de las comunidades objeto de estudio y solo el $27 \%$, mostró algún conocimiento sobre el cambio climático. Se aplicó la técnica del análisis ciudadano de la situación ambiental local, para que los comunitarios reunidos en fórum, relataran que acciones habían desarrollado para enfrentar la sequía. La aplicación de este método participativo constituyó un paso importante para impulsar, conformar y dinamizar espacios de concertación y participación ciudadana, en los cuales la ciudadanía como protagonista social, formula propuestas para el mejoramiento y prevención del deterioro del hábitat urbano.

Los comunitarios expresaron en el fórum que se había realizado medidas de adaptación colectivas como la construcción de cisternas para acumular el agua e individuales, como la adquisición de tanques o la construcción de ellos en los hogares, a través de los CDR (organización de masas con gran membresía), se recaudó financiamiento para la compra de motores de agua. Se dijo además que el gobierno municipal destinó recursos materiales y financieros para la distribución de agua de fuentes subterráneas y se inició la construcción del trasvase Este-Oeste, obra monumental que asegurará traer el agua del oeste de la provincia, zona con mayor disponibilidad de este

Soc. \& Nat., Uberlândia, 26 (2): 261-270, mai/ago/2014 
recurso. El fórum sirvió para visibilizar las medidas de adaptación efectuadas, pues los comunitarios no relacionaban estas con la adaptación al cambio climático.

Con las entrevistas cualitativas se pudo conocer que las personas no identificaban la sequía como amenaza, pues no existía una cultura del riesgo. En esta etapa se les enseñó a identificar las vulnerabilidades ante el cambio climático a través de la matriz de vulnerabilidad y capacidad. En las tres comunidades estudiadas las vulnerabilidades detectadas fueron: el deterioro de las redes de acueducto, lo que trae consigo el derrame constante del agua en las calles, con la consiguiente pérdida del preciado líquido, además el agua se distribuye por ciclos, los que generalmente se alargan demasiado y los depósitos colectivos e individuales se quedan secos, la no existencia de metro contadores, unido a la falta de conciencia ambiental, provocan el despilfarro. Otras vulnerabilidades están asociadas a las condiciones naturales que propician una mayor exposición al fenómeno de sequía, así como las limitaciones financieras. La comunidad de Alcides Pino, además de esas vulnerabilidades, posee zonas que se inundan en temporada de lluvia, pues las viviendas están construidas en el cauce del río.

El estado sicológico de la comunidad también determina su posición ante el cambio climático y sus posibilidades de adaptación, lo que exige contemplar factores como las opiniones, las actitudes y las aspiraciones. Una comunidad es sicológicamente más vulnerable si sus miembros tienen una actitud de resignación, pesimismo dependencia. Por el contrario, las capacidades comunitarias se ven reforzadas cuando priman la confianza en las propias posibilidades, el espíritu de lucha o metas sociales compartidas. De las tres comunidades estudiadas el Distrito Lenin mostró optimismo y entusiasmo al trabajar por la adaptación, mientras que la comunidad El Llano se mostró menos participativa y los de Alcides Pino fueron los que mostraron menos motivación, por supuesto que ello responde a las características socioeconómicas de cada una de ellas.

Al realizar un análisis participativo de las capacidades en las comunidades objeto de estudio, se develaron las siguientes:
- Infraestructura mínima para el abastecimiento del agua.

- Búsqueda de soluciones para mitigar la sequía por parte gobierno.

- Nivel educacional alto, lo que permite asimilar con facilidad nuevos saberes.

- Existencia de organizaciones barriales que funcionan como redes.

- Presencia de liderazgo de algunas personas que contribuyen a la movilización.

Con la identificación de las debilidades y las fortalezas de las comunidades, se estaba en condiciones de diseñar el programa de educación ambiental para la adaptación al cambio climático, el que se caracterizó por un diseño participativo. En la elaboración del programa se consideró que los espacios de aprendizaje deben constituirse como procesos donde exista real participación de los involucrados, lo que garantizaría que sus saberes y criterios sean considerados en la concepción y materialización de la educación ambiental aplicada.

Características y particularidades del diseño e implementación del programa de educación ambiental aplicado en tres comunidades de la ciudad de Holguín, Cuba

Las comunidades estudiadas están bien organizadas, con buen funcionamiento de las redes sociales, se ubican en los barrios: Distrito Lenin, El Llano y Alcides Pino, espacios con urbanización básicamente residencial y los servicios diarios asociados a las áreas de vivienda se encuentran servidos por infraestructuras técnicas, aunque el trazado vial resulta incompleto. La selección se realizó a partir de considerar la presencia de problemas urbano- ambientales vinculados con la variabilidad del clima. En la evaluación del medio ambiente urbano de la ciudad de Holguín efectuada en el período 2004-2008 (Agenda 21/GEO Holguín, 2008) se evidenció los problemas ambientales de las tres zonas. Alcides Pino concentraba la mayor cantidad de impactos ambientales de la ciudad, además de ser priorizado por el gobierno municipal al presentar dificultades sociales, Distrito Lenin y El Llano con similares características socio-ambientales, marcada por la escasez y contaminación del recurso agua e 
Experiencias en la aplicación de la educación ambiental como herramienta para la adaptación al cambio climático en espacios comunitarios, en Holguín-Cuba

inundaciones en períodos lluviosos pues las viviendas están construidas en el cauce de los arroyos.

La adaptación de los sistemas humanos es un proceso que demanda la participación de varios actores, por esta razón la educación ambiental se efectuó por grupos de edades: niñas y niños, jóvenes y adultos. La preparación de los más pequeños se realizó con el apoyo de la escuela, la que jugó un importante papel, los jóvenes fueron convocados por los estudiantes de la universidad, residentes en las comunidades, y los adultos a través de las organizaciones de masas. El programa ejecutado incluyó los conocimientos, el sistema de habilidades y valores así como la evaluación de la asimilación de los contenidos; se realizó sobre la base de una pedagogía conversacional y crítica, que implica un proceso de construcción de identidades con una fuerte carga ideológica y transformadora. El conocimiento difundido, ayudó a incorporar nuevos saberes sobre la temática abordada y el contexto tal y como es, este tipo de aprendizaje da la posibilidad de aprehender la riqueza y la complejidad de la realidad.

El conocimiento es favorecedor de la adaptación, pues la participación reflexiva en la construcción de saberes estimula el proceso del aprendizaje enseña cómo hay que actuar con respecto a problemas específicos, el desarrollo de competencias, permiten que los involucrados mejoren su entorno. Los contenidos estuvieron dirigidos al manejo adecuado y conservación de los componentes ambientales (agua, aire, suelo energía) y de los ecosistemas con los que se interactúa para reducir los impactos negativos como: la degradación, contaminación, desequilibrio, pérdida de la biodiversidad entre otros. Se argumentó que debe ser potenciada las producciones limpias, el empleo de fuentes renovables de energía, el tratamiento de residuos y el reciclaje, la protección y la rehabilitación de la biodiversidad. Las temáticas abordadas en las acciones educativas, estuvieron dirigidas a incorporar nuevos conocimientos sobre la base de los intereses y necesidades de las personas de la comunidad, los principales contenidos abordados se organizaron de la siguiente manera:

- El cambio climático, sus principales causas y consecuencias.

- Las vinculaciones entre la comunidad, su entorno regional, nacional y global.
- La vulnerabilidad y el riesgo asociado al cambio climático. Impacto en las condiciones de vida de la población.

- La contaminación ambiental por desechos sólidos, contribución de su acumulación al cambio climático.

- La legislación ambiental como instrumento de protección de los ecosistemas.

- La reforestación y su contribución a la mitigación de los efectos del cambio climático.

- La convivencia sostenible. Conductas y estilos de vida amigables con el medioambiente urbano.

- Medidas de adaptación al cambio climático en los hogares y el barrio.

Dieleman y Juárez (2008) también consideran importante ver y entender la realidad en formas tangibles, permitiendo aprender la riqueza y la complejidad del entorno. Al mismo tiempo, es importante conocer a través del análisis de los problemas específicos y elaborar soluciones. Esa fue una de las pretensiones del programa de educación ambiental aplicado, pues en él se establecieron los vínculos entre ser, hacer, conocer, pensar y sentir, pues los comunitarios no solo incorporaron conocimientos, además trabajaron en la transformación de su espacio comunitario, aportando beneficios ambientales.

Aprender haciendo fue uno de los principios aplicados en la puesta en práctica del programa, las habilidades se desarrollaron combinando actividades teóricas con acciones prácticas y se logró trabajar las que a continuación se exponen:

- Identificar problemas ambientales de la comunidad, definiendo las vulnerabilidades y capacidades.

- Describir el estado del medio ambiente en las comunidades y su relación con el cambio climático.

- Planificar acciones de adaptación al cambio climático, en los hogares y los espacios comunitarios, que contribuyan a atenuar o disminuir impactos sobre los medios de vida. 
- Socializar conocimientos y experiencias sobre el cambio climático a través de promotores comunitarios.

Las acciones de adaptación al cambio climático, dentro de un paradigma de sustentabilidad de carácter transformador, dependen de las actitudes de los participantes. Estas pueden caracterizarse por la apertura y compromiso, o el desinterés y la desmotivación. Es importante que las personas de la comunidad se vean involucradas en la problemática y en las transformaciones con un compromiso social de contribuir a la sostenibilidad. En este contexto resulta ineludible ocuparse de los valores y actitudes a través de la educación ambiental.

Las personas en su actuar diario se forman valores que constituyen los objetos de sus intereses y en lo concerniente a su conciencia desempeñan el papel de puntos de referencias diario en su actividad social. Y son los valores los que determinan las decisiones que se toman, también en relación con el manejo de nuestro medio ambiente en general. La educación ambiental debe demostrar eminentemente los valores colectivos, donde se incluyen todos los tipos de existencia, y no sólo la existencia humana, esta relación de lo colectivo con lo individual aporta una mejor compresión de la relación naturaleza - sociedad. Los valores desarrollados por el programa fueron:

- Respeto y cuidado al patrimonio natural y construido, lo que significa proteger los elementos naturales y construidos, deteniendo la deforestación, no contribuir a la contaminación arrojando desechos sólidos, una de la prácticas más cotidiana observada en las ciudades, entre otras conductas agresivas al medio ambiente.

- Mantener una posición equilibrada entre todo los componentes de la naturaleza, los seres humanos no somos el centro del universo, constituimos solo "... una mera hebra de la trama de la vida...”(CAPRA, 1998, p.29)

- La solidaridad y el humanismo, para apoyar a todas las personas que lo necesiten en las situaciones de desastre que el cambio climático puede generar.
- El respeto a los límites de la naturaleza considerando adecuadamente los aspectos económicos, ecológicos y sociales.

- La preocupación por el conocimiento del medio que lo rodea, fue abordado con particular énfasis, pues contribuye a ampliar la cultura ambiental y a la concientización que tanta falta hacen.

- La corresponsabilidad pues en la interacción con las comunidades se observó que muchos de los problemas que enfrentan pueden ser asumidos con una actitud corresponsable, considerando que esta no es más que la responsabilidad compartida, lo que significa que no solo el Estado y la instituciones deben solucionar los problemas del barrio, los pobladores pueden tomar una posición activa ante los mismos.

El programa de educación ambiental, incluyó la planificación en las comunidades, como un proceso que involucra a los actores locales, se realizó a través del diseño de acciones que contengan las medidas de adaptación al cambio climático, en búsqueda de alternativas en una dirección deseada, para ello se trazaron metas parciales a modo de escalonamiento en espiral; en la configuración de las actividades se consideraron los elementos históricos, geográficos y culturales necesarios para el cambio y evolución de las experiencias de los propios actores.

Se efectuaron acciones como: fórums comunitario para la identificación de los problemas ambientales relacionados con el cambio climático, debate de videos sobre cambio climático, charlas por el día mundial del aire puro, festival artístico para celebrar el Día Mundial del Medio Ambiente, higienización de las comunidades y recogida de materias primas para su clasificación y reutilización, debate sobre la importancia de los árboles, reforestación de patios y espacios públicos, actividades culturales con la participación de niños y niñas de la comunidad por el Día Internacional del Agua Dulce y talleres comunitarios sobre las acciones a realizar en el hogar para la adaptación al cambio climático. Las acciones planificadas, fueron básicamente de concientización se distinguieron por la transformación de saberes, se efectuaron en espacios 
Experiencias en la aplicación de la educación ambiental como herramienta para la adaptación al cambio climático en espacios comunitarios, en Holguín-Cuba

informales, en los que se multiplicaron conocimientos a partir de la construcción colectiva, lo que los ayudó a organizar el trabajo e involucrar a más personas en la protección del medio ambiente.

Se efectuó la evaluación del programa de educación ambiental para la adaptación al cambio climático de forma sistemática, permitió determinar el mérito de las actividades educativas con el propósito de mejorarlas. Además proporcionó datos e información relevante para la toma de decisiones y contribuyó a la participación de los diferentes actores, los que expresaron sus consideraciones de forma crítica.

La evaluación se realizó en todas las etapas del proceso, para ello se utilizaron elementos cualitativos básicamente de consenso para evaluar la pertinencia del diagnóstico y de la propuesta de acciones a realizar para eliminar o minimizar los problemas ambientales, así como la participación de los diferentes actores en las actividades convocadas y la calidad de las acciones de educación ambiental. Además se utilizaron indicadores cuantitativos como: cantidad de actividades de formación y número de participantes.

La educación ambiental estuvo dirigida a transferir conocimientos contextualizados con el propósito de desarrollar capacidades en los actores locales, estuvo dirigida a valorizar el conocimiento implícito en las prácticas efectuadas. Para evaluar la calidad de las mismas al concluir cada una de las actividades de formación se solicitó a los participantes que emitieran su juicio sobre la eficacia de estas y expresaron lo siguiente:

- Los temas que hemos recibido son de gran utilidad pues podemos aplicar lo aprendido cotidianamente.

- Los nuevos conocimientos que hemos incorporado, podemos multiplicarlos a través de nuestros promotores.

- Hemos aprendido que con la integración de varios actores podemos enfrentar mejor los problemas que se dan en las comunidades.

Los criterios aportados por los participantes sobre las acciones de formación, evidenciaron que las actividades se realizaron con calidad, pues permitieron mejorar las relaciones horizontales a través del diálogo de temas que resultan relevantes para la construcción colectiva de saberes. La evaluación de la participación de los actores en las diferentes actividades, se realizó de forma cualitativa para ello se consideraron las categorías: baja, moderada y alta, para valorar la motivación de los actores involucrados, se utilizaron las mismas categorías.

Para otorgar las categorías se efectuaron talleres, en el que los participantes a través de la técnica de las tarjetas emitieron sus criterios evaluativos, en el desarrollo del mismo se apeló por la justicia en las evaluaciones. La participación y motivación de los representantes del gobierno y las instituciones existentes en la comunidad fue evaluada de moderada, pues no se involucraron al nivel que demandaba la población, los niños y niñas, junto a los adultos de la tercera edad, fueron protagonistas en el proceso de formación, su participación y motivación fue considerada alta. Los jóvenes participaron de forma intermitente, en menor composición, la categoría otorgada para ellos fue baja, las personas adultas generalmente estaban presente, pero más pasivas, la evaluación otorgada fue moderada. En general la participación en las acciones de educación ambiental efectuadas, fueron consideradas moderadas, faltó masividad.

También se efectuó una evaluación cuantitativa de las actividades de formación sobre la base del análisis de la cantidad de actividades planificadas y las realizadas. En cinco años de implementación del programa se planificaron 65 actividades y se pudieron efectuar 57, lográndose el 95\% de cumplimento.

La evaluación de la implementación del programa de educación ambiental para la adaptación al cambio climático, con la participación de los comunitarios permitió:

- Registrar y analizar las experiencias (positivas y negativas) adquiridas.

- Retroalimentación de prácticas anteriores, propiciar aprendizajes.

- Determinar los niveles de desarrollo alcanzados.

- Valorar los procesos en su integridad.

- Obtener elementos de juicio fundamentados a fin de tomar decisiones que permitan orientar las acciones presentes y mejorar las futuras.

- Determinar si los cambios producidos se encuentran en correspondencia con las expectativas de los participantes. 


\section{CONSIDERACIONES FINALES}

La educación ambiental para el cambio climático requiere de un nuevo nivel de conciencia, ante el reto que impone la variabilidad del clima, las comunidades locales deberán asumir nuevos enfoques, las acciones de adaptación que se generen en estos contextos, ineludiblemente corresponden al desarrollo sostenible. La participación de los comunitarios ocupa un lugar relevante donde se estimula el aprendizaje colaborativo, el intercambio de saberes y la crítica en función de cambiar la realidad, la combinación del conocimiento formal con la experiencia basada en acciones, donde estas se realizan con el propósito de trasformar los espacios comunitarios a través de la solución o mitigación de los problemas ambientales.

Los programas de formación ambiental para la adaptación al cambio climático, incluyen la incorporación de aprendizajes sobre la identificación de vulnerabilidades y capacidades, en los hogares y en los espacios colectivos. La determinación de las debilidades, para adoptar una posición activa ante ellas y tratar de superarlas, la definición de las capacidades, permite saber el patrimonio que se posee para afrontar la adversidad y lograr la innovación que se necesita. Iniciar con el diagnóstico admite conocer de qué recursos humanos, sociales, físicos, naturales y financieros se disponen para la adaptación.

El programa de educación ambiental implementado incluyó la planificación de acciones, la que se efectuó con la integración entre los participantes, pues es clave para conseguir los objetivos propuestos, posibilita la construcción de nuevos estilos, contribuye a motivar la innovación y la solución de problemas antiguos con propuestas nuevas, es por ello que aprender haciendo es una de las características de la educación ambiental para la adaptación al cambio climático, donde se incluye la proyección de acciones a corto y mediano plazo, de forma participativa, con un análisis integral de las relaciones naturaleza - sociedad. El resultado de la planificación se vio materializado en las actividades ejecutadas, las que estuvieron dirigidas a lograr la resiliencia, como la capacidad de la comunidad de asimilar y recuperarse de los efectos de fenómenos meteorológicos extremos.

El programa aplicado en tres comunidades de la ciudad de Holguín, contribuyó a transformar el saber ambiental, al trabajar el cambio climático, no solo en el sentido de las exigencias en el manejo integral del medio ambiente, sino también en la aparición de una nueva ética estructurada esencialmente en nociones, conceptos y actitudes de convivencia armónica, responsabilidad y austeridad, respeto, equidad, sostenibilidad y solidaridad que favorezca la adaptación.

La evaluación del programa de educación ambiental resultó significativa, pues permitió determinar el valor o mérito de actividades ejecutadas. Ésta se realizó de forma sistemática encaminada a determinar objetivamente la calidad de la formación, la participación de la ciudadanía permitió conocer y tener los criterios necesarios para tomar decisiones con conocimiento de causa de los impactos positivos o no, que generaba el programa. En las tres comunidades en las que se implementó el programa, los participantes valoraron de relevantes las acciones realizadas y los objetivos fueron cumplidos. 


\section{REFERENCIAS}

ALDUNCE, P; NERI, C; SZLAFSZTEIN, C. Hacia la evaluación de prácticas de adaptación ante la variabilidad y el cambio climático. 2. ed. Belém: Numa-UFPA, 2008.

ANGENDA 21/GEO HOLGUÍN. Perspectivas del medio ambiente urbano. La Habana: Ed. Academia, 2008.

CARE INTERNACIONAL. Manual para el análisis de la capacidad y vulnerabilidad climática. Ed. CARE: Lima, 2010.

COMUNIDAD ANDINA. Articulando la gestión del riesgo y la adaptación al cambio climático en el sector agropecuario. Lima: Predecan, 2009.

DIELEMAN, H; JUÁREZ, M. ¿Cómo se puede diseñar educación para la sustentabilidad? Rev. Int. Contaminación Ambiental, México, v.24 n.3, p.131147, 2008.

CAPRA, F. La trama de la vida. Una nueva perspectiva de los sistemas vivos. 2. ed. Barcelona: Anagrama, 1998.

GRUPO INTERGUBERNAMENTAL DE EXPERTOS SOBRE EL CAMBIO CLIMÁTICO. Cuarto Informe de Evaluación, Ginebra, 2007. Disponible en: <http://www.ipcc.ch>, acceso dez. 2007.

LEVINE, T; ENCINAS, C. Adaptación al cambio climático: Experiencias en América Latina, Revista virtual REDESMA, v.2 n.3, p.25-32, 2008.

PNUD. La lucha contra el cambio climático: Solidaridad frente a un mundo divido. 1.ed. Nueva York: AcifeOgrady, 2008

ROLAC-UN HABITAT. Metodología para realizar Análisis Ciudadano de la Situación Ambiental Local, 2011 (mimeo).
SECRETARIA DE AMBIENTE Y DESARROLLO SUSTENTABLE. Manual de vulnerabilidad y adaptación al cambio climático para la gestión y la planifcación local. Buenos Aires: Ed. Carolina Nahón, 2010.

SFEIR-YOUNIS, A. Educación, economía ambiental y espiritualidad. Rev. de Estudios Sociales. Bogotá, n.32, p.241-254, 2009. 